\title{
Rendimiento académico y Trastorno de déficit de atención-hiperactividad en Escuelas de EI Progreso, Yoro, 2018.
}

Academic performance and attention deficit hyperactivity disorder in schools, of Progreso, Yoro, 2018.

\section{Nazaret Emisel Mendoza Contreras*, Luisa María Pineda**, Mario Roberto Velásquez ***}

\section{RESUMEN}

Antecedentes. El Trastorno de déficit de atención con hiperactividad (TDAH) define un patrón persistente de inatención y/o hiperactividad/impulsividad, incoherente con el nivel de desarrollo del individuo. Inicia en la infancia e interfiere en la adaptación, funcionamiento social y rendimiento académico. Objetivo. Determinar el rendimiento académico en los escolares asociado a trastorno de déficit de atención con hiperactividad en escuelas públicas de El Progreso, Yoro durante el período de enero a junio 2018. Pacientes y métodos. Estudio descriptivo transversal analítico. Universo de 12,440 escolares de escuelas públicas. Se estimó un tamaño muestral de $300 \quad(10.8 \%$, IC95\%). Se incluyeron escolares de 6-12 años. Se excluyeron patologías congénitas o adquiridas con afectación directa en personalidad/conducta, fracaso escolar secundario a estresores ambientales. Las variables incluyeron datos del escolar/ padre/tutor, rendimiento académico, antecedentes familiares/personales patológicos. Se utilizó el test de Conners para padres/maestros, así como el cuestionario DSM-V para diagnóstico TDAH. La información recolectada fue ingresada en Epiinfo versión 7.2.2.6 (CDC, Atlanta). Los resultados se presentan como frecuencias, porcentajes, valor de P, OR y $\mathrm{chi}^{2}$. La información personal

* Médico Residente tercer año de Pediatría, UNAH-VS

** Epidemióloga pediatra HMCR

*** Médico especialista en Pediatría HMCR

Dirigir correspondencia a: nmendoza227@hotmail.com

Recibido: 10 de Julio de 2018 Aprobado: 20 Septiembre de 2018 de los casos se manejó confidencialmente. Resultados. La prevalencia de TDAH fue del 36\% (108/300). Se encontró asociación estadísticamente significativa $(P<0.05)$ en la edad del escolar, estado civil del padre/ tutor, antecedentes familiares, persona que vive con él. El rendimiento escolar fue satisfactorio $\leq 70-80 \% \quad(53 \%)$. No hubo diagnóstico de TDAH en 64\%. Conclusión. Es importante realizar una intervención oportuna e integral para prevenir las complicaciones del TDAH y la afectación directa en el rendimiento académico de los escolares.

PALABRAS CLAVE: Rendimiento Académico, Trastorno por Déficit de Atención con Hiperactividad, Pediatría.

\section{ABSTRACT}

Attention deficit hyperactivity disorder (ADHD) defines a persistent pattern of inattention and/or hyperactivity/impulsivity, incoherent with the individual's level of development. It begins in infancy and interferes with adaptation, social functioning and academic performance. Objective. To determine academic performance in schoolchildren associated with attention deficit hyperactivity disorder in public schools in $\mathrm{El}$ Progreso, Yoro during the period from January to June 2018. Patients and methods. Analytical cross-sectional descriptive study. Universe of 12,440 public school students. 
A sample size of 300 was estimated $(10.8 \%$, IC95\%). Schoolchildren aged 6-12 years were included. Congenital or acquired pathologies with direct affect on personality/ behavior, school failure secondary to environmental stressors were excluded. The variables included data on schoolchildren/parents/guardians, academic performance, family history/ personal pathology. The Conners test for parents/teachers was used, as well as the DSM-V questionnaire for ADHD diagnosis. The information collected was entered into Epiinfo version 7.2.2.6 (CDC, Atlanta). The results are presented as frequencies, percentages, value of $\mathrm{P}, \mathrm{OR}$ and chi2. Personal case information was handled confidentially. Results The prevalence of ADHD was $36 \%$ (108/300). A statistically significant association $(P<0.05)$ was found in the age of the schoolchild, marital status of the parent/guardian, family history, person living with the schoolchild. School performance was satisfactory $\leq 70-80 \%$ $(53 \%)$. There was no diagnosis of ADHD in $64 \%$. Conclusion. It is important to perform a timely and comprehensive intervention to prevent the complications of ADHD and the direct impact on the academic performance of schoolchildren.

KEYWORDS: Academic Performance, Attention Deficit Disorder with Hyperactivity, Pediatrics.

\section{INTRODUCCIÓN}

Actualmente en las escuelas públicas del estado de Honduras, los escolares podrían presentar patrones persistentes de inatención y/o hiperactividad/impulsividad, que resultan no acordes con el nivel de desarrollo. El no intervenir de manera oportuna en esta patología desatendida podría presentar consecuencias que afecten de forma directa el rendimiento académico de los escolares, y sobre todo, conlleva a un aumento de la deserción escolar; por el hecho de asociar a estos con mala conducta, o disminución en su rendimiento académico, de manera que el fracaso y abandono escolar, las conductas adictivas y los problemas relacionados a la justicia se encuentran entre los resultados más preocupantes asociados a TDAH y su carácter crónico hace que el problema persista en el periodo de la adolescencia y adultez.

El Trastorno de déficit de atención con hiperactividad (TDAH) define un patrón persistente de inatención y/o hiperactividad/impulsividad, incoherente con el nivel de desarrollo del individuo. El trastorno tiene un inicio en la infancia e interfiere en la adaptación, funcionamiento social, rendimiento académico y ocupacional del paciente. ${ }^{[1,2]}$ Se considera como un trastorno de la función cerebral en niños, adolescentes y adultos; asimismo, se caracteriza por la presencia persistente de síntomas comportamentales y cognoscitivos (Pineda, 1999)..[3] La aptitud escolar, desempeño o rendimiento académico (R.A) es entendido como el sistema que mide los logros y la construcción de conocimientos en los estudiantes, los cuales se crean por la intervención de didácticas educativas que son evaluadas a través de métodos cualitativos y cuantitativos en una materia[4] La incidencia del TDAH no está claramente establecida, aunque los estudios más recientes consideran que alrededor de un $5-10 \%$ de los niños de la población mundial presentan TDAH, y de estos, un $15-50 \%$ mantendrán el diagnóstico en la edad adulta. [5] En Latinoamérica existen al menos 36 millones de personas con TDAH y menos de un cuarto de los pacientes se encuentran bajo tratamiento multimodal; de los cuales el $23 \%$ tiene apoyo terapéutico psicosocial y el $7 \%$ tratamiento farmacológico adecuado. ${ }^{[6]}$ 
Por parte del postgrado de pediatría, no existe un estudio orientado al TDAH en escolares y tener un dato epidemiológico de esta temática permite conocer el grado de afectación y la magnitud de sus consecuencias en nuestra población de estudio, por lo que el objetivo de este estudio fue Determinar el rendimiento académico en escolares asociado a trastorno de déficit de atención con hiperactividad en escuelas públicas de El Progreso, Yoro durante el período de enero a junio 2018.

\section{PACIENTES Y MÉTODOS}

Se realizó un estudio descriptivo transversal con enfoque cuantitativo. El universo estuvo constituido por 12,440 escolares de escuelas públicas del municipio de El Progreso, Yoro, pertenecientes al distrito escolar 22 en jornada matutina, durante el período enero a junio 2018. El muestreo fue aleatorio seleccionando una muestra de 300 alumnos de 4 escuelas públicas (Jaime $O$ 'leary, Dos de Marzo, Pedro Pascual Amaya, San José) con edades de 6-12 años cuyos padres/encargados/tutores/maestros hayan firmado consentimiento informado aceptando participar en el estudio. Se excluyeron escolares con patologías congénitas $\mathrm{o}$ adquiridas que afectaron directamente la personalidad y conducta, preescolares menores de 6 años y preadolescentes mayores de 12 años, así como fracaso escolar secundario a estresores ambientales, trastornos sensoriales importantes, así como trastornos endocrinológicos, metabólicos y nutricionales adquiridos. Los datos fueron recolectados mediante instrumento de recolección que incluyó: identificación del escolar, rendimiento académico, datos del padre/tutor, antecedentes familiares, núcleo intrafamiliar, antecedentes personales patológicos, escala de Conners para padres/profesores y el cuestionario del DSM-V para diagnóstico de TDAH.
El procesamiento y tabulación de los datos se realizó mediante el paquete estadístico Epiinfo versión 7.2.2.6 (CDC Atlanta), presentándose estos como frecuencias, porcentajes, rangos, promedios, OR $\mathrm{chi}^{2}$ y valor $\mathrm{P}$. La información personal de los casos se manejó confidencialmente.

\section{RESULTADOS}

A continuación, se presentan los resultados de los escolares que presentaron diagnóstico de TDAH durante el periodo enero - junio 2018 en escuelas públicas del municipio de El Progreso, Yoro. Los resultados se presentan en base a 300 .

\section{A. Prevalencia de TDAH en escolares}

Gráfico 1. Prevalencia de TDAH según variantes en escolares estudiados en El Progreso, Yoro 2018

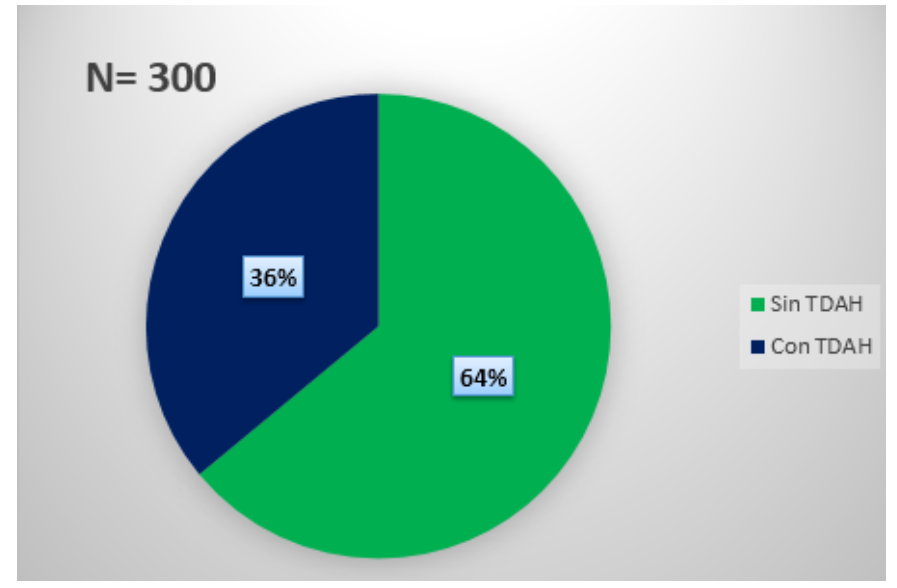

Se seleccionaron un total de 4 escuelas públicas: Jaime O'leary, Dos de Marzo, Pedro Pascual Amaya, San José, para un total de 2,754 alumnos, encontrando una prevalencia de Trastorno de Déficit de Atención e Hiperactividad de 36\% (108/300).

B. Características sociodemográficas de los escolares con TDAH. La edad escolar más frecuente fue 10 años 24 (22.2\%), predominó el género masculino $78(72.2 \%)$, la procedencia fue urbana en el total de los casos 108 (100\%), $106(98.1 \%)$ no repitieron año escolar, el estado 
civil del padre/tutor más frecuente fue unión libre $67(62.0 \%)$ y el ingreso mensual mayormente encontrado fue menor L. 9,000.00 104 (96.3). Se encontró asociación estadísticamente significativa en la edad y estado civil $(P<0.05)$ (Tabla 1$)$.

\section{Anexos}

Tabla 1. Características sociodemográficas de los escolares con TDAH. $\mathrm{N}=108$

\begin{tabular}{|c|c|c|c|c|}
\hline CARACTERISTICA & N (\%) & $\mathrm{Chi}^{2}$ & df & Valor $\mathrm{P}$ \\
\hline \multicolumn{5}{|l|}{ Edad (años) } \\
\hline 6 & $10(9.3)$ & & & \\
\hline 7 & $20(18.5)$ & & & \\
\hline 8 & $18(16.7)$ & & & \\
\hline 9 & $17(15.7)$ & 40.1232 & 21 & $0.0061^{\wedge}$ \\
\hline 10 & $24(22.2)$ & & & \\
\hline 11 & $9(8.3)$ & & & \\
\hline 12 & $10(9.3)$ & & & \\
\hline \multicolumn{5}{|l|}{ Sexo } \\
\hline Hombre & $78(72.2)$ & 26.9996 & 3 & 0 \\
\hline Mujer & $30(27.8)$ & & & \\
\hline \multicolumn{5}{|l|}{ Procedencia } \\
\hline Urbana & $108(100.0)$ & 1.1326 & 3 & 0.7692 \\
\hline Rural & $0(0.0)$ & & & \\
\hline \multicolumn{5}{|l|}{ Repitencia de ańo escolar } \\
\hline No & $106(98.1)$ & 3.3621 & 3 & 0.3391 \\
\hline Si & $2(1.9)$ & & & \\
\hline Estado civil del padre/tutor & & 30.2327 & 12 & $0.0026^{*}$ \\
\hline Unión libre & $67(62.0)$ & & & \\
\hline Casado & $12(11.1)$ & & & \\
\hline Soltero & $29(26.9)$ & & & \\
\hline \multicolumn{5}{|l|}{ Ingreso económico mensual } \\
\hline$<$ L. $9,000.00$ & $104(96.3)$ & 2.2838 & 3 & 0.5156 \\
\hline$>$ L. $9,000.00$ & $4(3.7)$ & & & \\
\hline
\end{tabular}

C. Antecedentes familiares y personales patológicos.98 (90.7\%) no presentaron antecedentes familiares con TDAH, 7 (6.4\%) presentaron otros familiares con antecedente de TDAH $(P=0.0026), 35 \quad(32.4 \%)$ de los escolares vivían con ambos padre y madrepadrastro $(P=0.0271), \quad 63(58.3 \%)$ no tenía antecedente de desintegración familiar, 39 (36.2\%) tenían dos hermanos, 13 (12\%.0) presentó antecedente personal patológico de dermatitis atópica, y $2(1.8 \%)$ presentaron alteraciones del sueño (ronquido) (Tabla 2).

Tabla 2. Antecedentes familiares y personales patológicos de los escolares con TDAH. $\mathrm{N}=108$

\section{Asociación multivariada con TDAH}

El gráfico 1 muestra la relación entre TDAH y rendimiento académico, encontrándose que la variante clínica más frecuente de TDAH fue la presentación predominante con falta de atención en 34 escolares con rendimiento satisfactorio. El gráfico 2 muestra la relación entre TDAH y la edad. El gráfico 3 muestra la relación entre antecedentes personales patológicos y TDAH.

\begin{tabular}{|c|c|c|c|c|}
\hline CARACTERISTICA & $\mathrm{N}(\%)$ & $\mathrm{Chi}^{2}$ & df & Valor $\mathrm{P}$ \\
\hline \multicolumn{5}{|l|}{ Antecedentes familiares TDAH } \\
\hline No & $98(90.7)$ & 13.9695 & 3 & $0.0029^{*}$ \\
\hline & $10(9.3)$ & & & \\
\hline \multicolumn{5}{|l|}{ Familiares con antecedente TDAH } \\
\hline Madre & $0(0.0)$ & 0.5801 & 3 & 0.901 \\
\hline Padre & $0(0.0)$ & 0.5801 & 3 & 0.901 \\
\hline Hermanos & $3(2.8)$ & 6.9604 & 3 & 0.0732 \\
\hline Otros $^{*}$ & $7(6.4)$ & 14.2729 & 3 & $0.0026^{*}$ \\
\hline \multicolumn{5}{|l|}{ El escolar vive con } \\
\hline Ambos padres & $35(32.4)$ & & & \\
\hline Madre y padrastro & 35 (32.4) & & & \\
\hline Su madre & $18(16.7)$ & & & 00271 * \\
\hline Padre y madrastra & $11(10.2)$ & 31.2212 & 18 & $0.0277^{\wedge}$ \\
\hline Otros & $6(53.6)$ & & & \\
\hline Su padre & $3(2.8)$ & & & \\
\hline Algún pariente & $0(0.0)$ & & & \\
\hline \multicolumn{5}{|l|}{ Desintegración familiar } \\
\hline No & $63(58.3)$ & 11.7163 & 3 & 0.0084 \\
\hline \multirow{2}{*}{\multicolumn{5}{|c|}{ Número de hermanos }} \\
\hline & & & & \\
\hline Un hermano & $35(32.4)$ & & & \\
\hline Dos hermanos & $39(36.2)$ & 4.3245 & 9 & 0.8888 \\
\hline Hijo único & $17(15.7)$ & & & \\
\hline Tres o más hermanos & $17(15.7)$ & & & \\
\hline \multicolumn{5}{|l|}{$\begin{array}{l}\text { Antecedentes médicos/personales } \\
\text { patológicos }\end{array}$} \\
\hline $\begin{array}{l}\text { patologicos } \\
\text { Dermatitis atópica }\end{array}$ & $13(12.0)$ & 4.557 & 3 & 0.2073 \\
\hline Asma bronquial & $7(6.5)$ & $\begin{array}{l}4.551 \\
0.493\end{array}$ & 3 & 0.9204 \\
\hline Alergia & $9(8.3)$ & 3.8646 & 3 & 0.2765 \\
\hline Hipertensión arterial & $0(0.0)$ & 0 & 0 & 0 \\
\hline Diabetes mellitus & $0(0.0)$ & 0 & 0 & 0 \\
\hline Dislipidemia & $0(0.0)$ & 0 & 0 & 0 \\
\hline Enfermedad celiaca & $0(0.0)$ & 0 & 0 & 0 \\
\hline Otros & $0(0.0)$ & 0 & 0 & 0 \\
\hline \multicolumn{5}{|l|}{ Alteraciones del sueño } \\
\hline Ronquido & $2(1.8)$ & 0.3651 & 3 & 0.9474 \\
\hline Miedo a la oscuridad & $0(0.0)$ & 1.1094 & 3 & 0.7748 \\
\hline Enuresis & $0(0.0)$ & 0.5644 & 3 & 0.9045 \\
\hline Múltiples despertares & $0(0.0)$ & 0.5644 & 3 & 0.9045 \\
\hline Enuresis & $0(0.0)$ & 0 & 0 & 0 \\
\hline Pesadillas & $0(0.0)$ & 0 & 0 & 0 \\
\hline Miedo a la oscuridad & $0(0.0)$ & 0 & 0 & 0 \\
\hline Ronquido & $0(0.0)$ & 0 & 0 & 0 \\
\hline Síndrome de apnea-hipoapnea del sueño & $0(0.0)$ & 0 & 0 & 0 \\
\hline Otros & $0(0.0)$ & 0 & 0 & 0 \\
\hline
\end{tabular}

\section{Grafico 2.}

Diagnostico TDAH según variantes clínicas en escolares estudiados en El Progreso, Yoro 2018

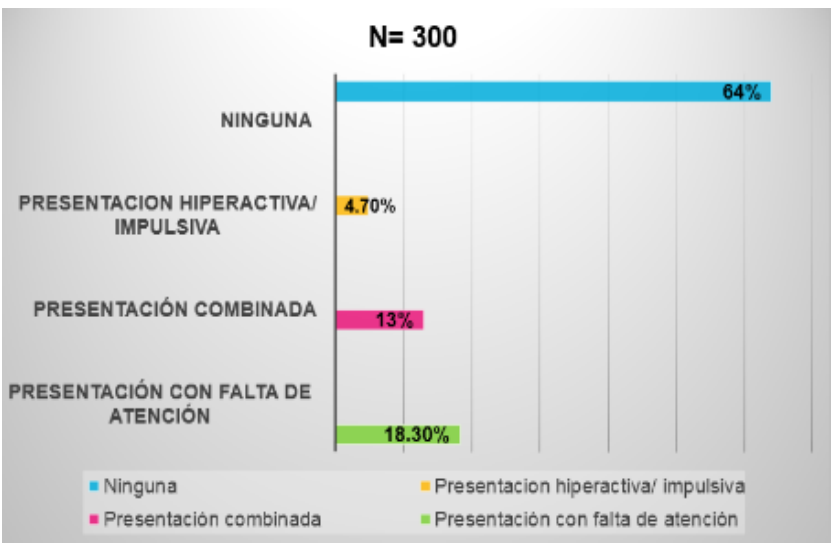

\section{Grafico 3.}

TDAH según edad en escolares estudiados en El Progreso, Yoro, 2018 


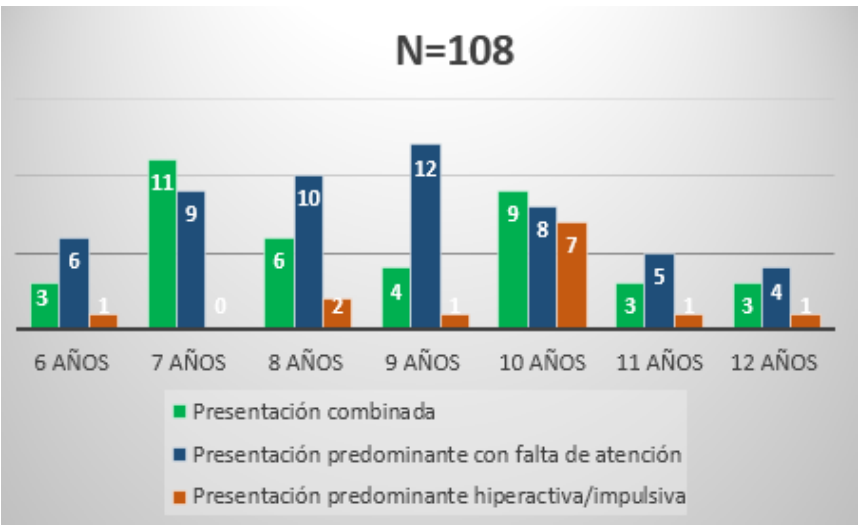

Grafico 4. Rendimiento académico en escolares estudiados con TDAH en El Progreso, Yoro, 2018

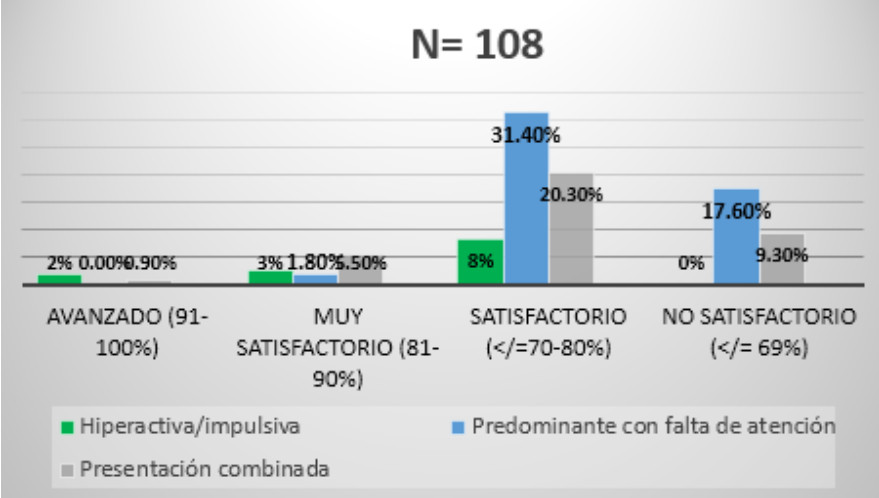

\section{DISCUSIÓN}

El trastorno por déficit de atención e hiperactividad (TDAH) es un problema de inicio en la infancia que comprende patrones persistentes de falta de atención, hiperactividad e impulsividad que pueden continuar a lo largo de la vida e influir en los resultados funcionales, tanto en las relaciones en la escuela como en las actividades cotidianas. [7] El TDAH es uno de los trastornos más frecuentes en la infancia. En este sentido, si bien las tasas de prevalencia del trastorno según los diferentes estudios irían desde el 1.5 al 18\% (Cardo et al., 2011), quizá el dato más aceptado en este sentido es el que proporciona el DSM-IV-TR (APA, 2002), según el cual entre el $3 \%$ y $7 \%$ de los niños en edad escolar presentarían este trastorno, diagnosticándose aproximadamente tres veces más en niños que en niñas. [8-10] Otro estudio realizado por Livia Segovia J., et al., en Lima, evidenció una prevalencia de $16,5 \%$, con predominio del género masculino. ${ }^{111]}$ Datos que contrastan a los reportados en el presente estudio, donde la prevalencia en escolares con TDAH fue del $36 \%$ respectivamente, y encontrándose más frecuentemente en el género masculino.

Pineda Santos M.A., realizó un estudio en el año 2016 sobre trastorno de déficit de atención e hiperactividad en las escuelas de San Pedro Sula, con el objetivo de determinar la prevalencia en población escolar, la metodología utilizada fue estudio descriptivo, cuantitativo de corte longitudinal, en 9 escuelas del distrito 21 y 22 en la jornada matutina, obteniendo una muestra de 235 escolares. Se encontró que el 51\% presentaron sospecha de TDAH mediante test de Conners aplicado a padres, y una prevalencia del $5.8 \%$. La variante clínica más frecuente fue de tipo combinada en $49 \%$, el género más frecuente fue el masculino con $75 \%$, y el grado con mayor afectación fue el tercero con 25\% respectivamente [12]

Se han realizado múltiples revisiones bibliográficas en referencia a la temática de TDAH enfocada en el adulto, uno de ellos por Reyes Ticas J.A.\& Cols., en el año 2010 [13], así como de comorbilidad entre TDAH y epilepsia, realizado en el 2010 por BarragánPérez E \& Cols., (6) Asimismo, Honduras formó parte del primer consenso Latinoamericano y declaración de México para el trastorno de déficit de atención e hiperactividad en LA, en donde un grupo de investigadores latinoamericanos intercambió el conocimiento y experiencia generada en distintos países de LA en relación del TDAH[14] 
Hay datos clínicos que señalan la alta prevalencia del TDAH en población infantil y adulta en España (Catalá-López et al., 2012), como en otros países modernos según estimaciones llevadas a cabo en las últimas tres décadas (Polanczyk, Willcutt, Salum, Kieling y Rohde,2014). ¿A qué puede atribuirse una prevalencia tan elevada? ¿A su comorbilidad con otras patologías (v. gr., trastorno de oposición desafiante)? ¿A la dificultad inherente a su diagnóstico? Dada su gran repercusión en el entorno sociofamiliar y escolar, parece razonable identificar los factores causales subyacentes (Gómez-Maqueo, Heredia y Mancona, 2014); otros autores se han interrogado también por el efecto de la utilización de diferentes guías a la hora de tratar el TDAH (Rabito-Alcón y Correas-Lauffer,2014). Con todo, dado el abordaje multimodal del TDAH, la respuesta no puede inferirse sólo de la aplicación desigual de tales guías, sino de la influencia de otros factores intervinientes en el proceso diagnóstico, entre los que se han formulado: 1) elementos arte-factuales, esto es, debido al uso de distintos instrumentos y de criterios diagnósticos del TDAH, como por ejemplo diagnosticarlo sólo cuando se manifiesta en un determinado contexto, como la escuela, mientras otros autores consideran imprescindible que se manifieste también en otros entornos; 2) razones de índole social, con el argumento de que ya desde 1990 la APA (Asociación Psicológica Americana) constata la elevada comorbilidad del TDAH con otros trastornos (v. gr., de conducta), con el requisito de que los niños afectados estén integrados en su entorno educativo, aplicándoseles las pautas psicoeducativas adaptadas a sus necesidades educativas especiales; 3) presión de las grandes empresas farmacéuticas para la prospección de nuevos casos, al estar en juego la prescripción de fármacos como el metilfenidato (Nolen-Koesksema, Fredickson, Loftus y Wagenaar, 2009). [15]
Los criterios vigentes para el diagnóstico del TDAH de acuerdo a la Asociación Americana de Psiquiatría, se pueden resumir de la siguiente manera: la presencia de al menos seis síntomas (de nueve posibles) de inatención y/o de seis síntomas (de nueve posibles) de hiperactividad/impulsividad, que están presentes por al menos seis meses en un grado que no concuerda con el nivel del desarrollo y que afectan las actividades sociales y académicas/ laborales. Deben ocurrir al menos algunos de estos síntomas antes de los doce años, estar presentes en dos o más contextos ( $p$. ej. en casa, escuela o trabajo, amigos o parientes), y no explicarse mejor por otro trastorno mental. [16] Existen tres subtipos según la última clasificación del DSM-V: predominio inatento, predominio hiperactivo-impulsivo y, el más común de ellos, el subtipo combinado. [17] Datos que se presentaron en la población de estudio, encontrando que la variante clínica más frecuente fue la presentación con predominio de falta de atención (18.3\%), seguido de la presentación combinada (13.0\%) e hiperactiva/impulsiva (4.7\%) respectivamente.Para el año 2010, Duarte Z \& Cols., realizaron un estudio de prevalencia de TDAH, relación con reprobación y estado nutricional en población escolar del Distrito Central, dichas edades estaban entre 6 a 13 años, tomando una muestra de 600 escolares de 10 centros educativos de forma aleatoria, y utilizando la escala de detección del TDAH, encontraron una prevalencia de $11.73 \%$, relación de sexo masculino-femenino $2: 1$ siendo la mayoría del 4to grado escolar. El subtipo de TDAH fue el mixto (inatentohiperactivo/impulsivo) y el índice de reprobación fue del $24.6 \%$ asociado a TDAH [18] 
Los niños que padecen TDAH han mostrado tener un déficit de las funciones ejecutivas, primordialmente la atención, memoria de trabajo, flexibilidad cognitiva y el control inhibitorio; además, manifiestan dificultades en la concentración organización, planificación, regulación del esfuerzo y el manejo de las emociones. El rendimiento en tareas de fluidez verbal es bajo y los tiempos de reacción son más lentos, con una percepción del paso de tiempo diferente a los niños sin TDAH. [19]

La hiperactividad, inatención e impulsividad son consideradas síntomas primarios del trastorno, y suelen ir acompañadas por dificultades cognitivas, emocionales, académicas e interpersonales que, a su vez, se constituyen en síntomas secundarios del desorden (Korzaniowsk \& Ison, 2013). De este modo, se convierten en uno de los principales motivos de consulta debido a las dificultades que generan en el rendimiento escolar, en conducta e interacción sociofamiliar, y en ocasiones afectan la autoestima (Rubiales, Bakker, Delgado, 2011). [17] pese a que el TDAH influye directamente en el rendimiento académico, los resultados de este estudio evidenciaron que el $56.4 \%$ de los escolares, presentaron un rendimiento académico satisfactorio (70-80\%)El trastorno por déficit de atención con hiperactividad (TDAH) persiste hasta la adolescencia y la vida adulta, y se encuentra frecuentemente asociado a otros problemas y trastornos comórbidos que conjuntamente presentan repercusiones negativas a lo largo del ciclo vital (Faraone, Biederman, \& Mick, 2006). Uno de los aspectos que está recibiendo un interés creciente se refiere a la identificación de los factores de tipo genético, biológico, familiar, personal, social y de diversa índole que pueden estar modulando el curso del trastorno. El estudio más exhaustivo sobre este tema lo han proporcionado los trabajos longitudinales, demostrando que, a pesar de ser un trastorno con una fuerte carga genética, los factores de tipo ambiental también contribuyen en su evolución. En este sentido, Cherkasova, Sulla, Dalena, Pondé y Hechtman (2013) realizaron una revisión sobre los factores que influyen en el curso del TDAH. Estos autores concluyeron que existen diferentes predictores según el periodo de desarrollo en el que se encuentre el sujeto: mientras que en el desarrollo temprano, cobran mayor importancia los factores genéticos y de adversidad prenatal; en los años escolares, la gravedad de la sintomatología de TDAH, el funcionamiento cognitivo, los factores familiares y las comorbilidades son los principales predictores de la persistencia del TDAH en la adolescencia, edad adulta y del ajuste en el funcionamiento a largo plazo. [20]

Los factores genéticos han estado fuertemente implicados en la etiología del TDAH. Se trata de un desorden multifactorial con una heredabilidad estimada alrededor del 76\%. En este sentido, se ha evidenciado que los hijos de un progenitor con TDAH tienen un 50\% de probabilidad detener los mismos síntomas. Cuando se evalúa a niños seleccionados a partir de padres con diagnóstico de TDAH de inicio infantil, se demuestra que el $57 \%$ de estos niños cumple con criterios de TDAH y de estos, un $75 \%$ ha estado en tratamiento. Smalley et al. encontraron que cuando se diagnostica a niños con TDAH y conductas oposicionistas a través del DSM-V, existe asociación significativa con un padre afectado en el $55 \%$ de los casos, siendo mayor para las niñas en un $63 \%$ en comparación con los niños. Sin embargo, no hay que menosprecar el papel del ambiente 
en la generación y el mantenimiento del trastorno a lo largo de la vida. Hay que anotar que es posible que cuanto más severo sea el trastorno en los niños, existe mayor probabilidad de tener un progenitor con TDAH. [21] En el presente estudio vista de ello, los resultados demostrados en este estudio encontraron correlación estadísticamente significativa entre el antecedente familiar directo de TDAH o de consanguinidad de primer grado (hermanos), así como el cuidador o tutor encargado y la presencia de desintegración familiar.

Los niños con TDAH presentan una alta incidencia de comorbilidad, pudiendo afectar hasta un $66 \%$ de los pacientes en algún momento de su vida, siendo el trastorno negativista desafiante, presente en un $40 \%$ de los niños con TDAH, el más frecuente; seguidos de los trastornos por ansiedad, con un $34 \%$, trastornos de la conducta en un $14 \%$, y los tics en un $11 \%$.

[22] Son múltiples los estudios

que asocian el TDAH con patología alérgica: asma, rinitis alérgica, dermatitis atópica, conjuntivitis alérgica, etc. En una revisión reciente se concluye que el TDAH se asocia de forma positiva con el asma, aunque se precisan más estudios para dilucidar la etiopatogenia de esta asociación. La posible conexión entre dermatitis atópica y TDAH fue descrita por primera vez por Schmitt et al en un estudio poblacional de 600.000 personas procedentes de Alemania, que, posteriormente, corroboraron en una revisión sistemática de 20 estudios poblacionales. Desde entonces, la asociación independiente entre la dermatitis atópica y TDAH se ha visto confirmada en varios estudios $\mathrm{y}$, más recientemente, en una actualización de la dermatitis atópica. Chou et al publicaron una prevalencia de rinitis alérgica del $28,4 \%$ y del $9,6 \%$ para asma en pacientes con TDAH. Aunque no hay datos sólidos en cuanto a la cifra de prevalencia de esta asociación, todos los autores coinciden en que la comorbilidad de varios trastornos alérgicos aumenta el riesgo de TDAH. ${ }^{23]}$ aunque no se encontró asociación estadísticamente significativa entre estas comorbilidades en los escolares estudiados, los datos demostraron que, si coexistía la presencia de asma bronquial, seguido de alergia y por último dermatitis atópica. La relación entre el sueño y el TDAH es conocida desde hace décadas y esta interrelación es compleja, multifactorial y multidireccional, de manera que los niños con alteraciones del sueño, con un sueño inadecuado o una mala calidad del mismo, desarrollan síntomas semejantes al TDAH. Los trastornos de sueño, muy frecuentes en los niños con TDAH, aparecen en un $25-50 \%$ de ellos y son 5 veces más frecuentes que en niños sanos. Las principales comorbilidades del TDAH y el sueño están frecuentemente asociadas con el ronquido $(55.7 \%)$ (más de la mitad de la noche), enuresis, dificultad para mantener el sueño (63\%) (más de dos despertares nocturnos), resistencia al acostarse y trastorno por movimientos rítmicos (61.5\%). [22] según los resultados en este estudio, la única alteración del sueño encontrada en los escolares fue la presencia de ronquido en un $2.6 \%$, dato que contrasta a lo mostrado en la literatura, al presentarse como principales el insomnio inicial, mantenimiento e incremento de la latencia de sueño seguido de múltiples despertares.

CONCLUSIÓN: Es importante realizar una intervención oportuna e integral para prevenir las complicaciones del TDAH y la afectación directa en el rendimiento académico de los escolares.

CONFLICTO DE INTERÉS: Los autores declaran que no existe conflicto de interés en la publicación del presente artículo. 


\section{REFERENCIAS BIBLIOGRÁFICAS}

1. Cunill R, Castells X. Trastorno por déficit de atención con hiperactividad. Med Clin (Barc). $2014 . \quad$ Disponible en: http://dx.doi.org/10.1016/ j.medcli.2014.02.025

2. Guerro-Prado D, et al. Evolución del estrés familiar en niños con trastorno por déficit de atención con hiperactividad. An Pediatr (Barc). 2015. Disponible en: http://dx.doi.org/10.1016/ j.anpedi.2014.12.004

3. Livia Segovia J, Ortiz Morán M, Vásquez Vega J. Prevalencia del trastorno por déficit de atenciónhiperactividad en escolares de una zona urbano-marginal de Lima. PsiqueMag. 2017; 6(1): 65-71. http://ojs.ucvlima.edu.pe/ index.php/psiquemag/article/view/173/91

4. Erazo O.A. El rendimiento académico, un fenómeno de múltiples relaciones y complejidades. Rev Vang Psicol. 2012; 2(2): pp 144-173. Archivo disponible en: https:// dialnet.unirioja.es/servlet/articulo? codigo $=4815141$

5. Quintero J, Castaño de la Mota C. Introducción etiopatogenia del trastorno por déficit de atención e hiperactividad (TDAH). Pediatr Integral 2014; XVIII (9): 600-608. Disponible en: https:// www.pediatriaintegral.es/wp-content/ uploads/2014/xviii09/02/ n9-600-608_Javier\%20Quintero.pdf
6. Palacio Ortiz J.D, De la Peña Olvera F, Barragán Pérez E. Declaración de Cartagena para el Trastorno por Déficit de Atención con Hiperactividad (TDAH): Un compromiso para todos. Rev Med Hondur. 2010; 78(3): 142-144. Disponible en: http:// www.bvs.hn/RMH/pdf/2010/pdf/ Vol78-3-2010-9.pdf

7.Catalá-López F, Hutton B. El tratamiento del trastorno por déficit de atención e hiperactividad en niños y adolescentes: epidemiología, multimorbilidad y servicios de salud integrados. An Pediatr (Barc). 2018;88(4):181 - 182 . https:// doi.org/10.1016/j.anpedi.2017.12.015

8. García T, González-Castro P, Rodríguez Pérez C, Cueli M, Álvarez García D, Álvarez $\mathrm{L}$. Alteraciones del funcionamiento ejecutivo en el trastorno por déficit de atención con hiperactividad y sus subtipos. Piscología Educativa. 20 (2014) 23 - 32. http://dx.doi.org/10.1016/j.pse.2014.05.003

9. Rivas-Juesas C, de Dios J.G, BenacPrefaci M, Colomer-Revuelta J. Análisis de los factores ligados al diagnóstico del trastorno por déficit de atención e hiperactividad en la infancia. Neurología. 2017;32(7):431 - 439. http:// dx.doi.org/10.1016/j.nrl.2016.01.006

10.Guerro-Prado D, et al. Evolución del estrés familiar en niños con trastorno por déficit de atención con hiperactividad. An Pediatr (Barc). 2015. http://dx.doi.org/10.1016/ j.anpedi.2014.12.004

11. Livia Segovia J, Ortiz Morán M, Vásquez Vega J. Prevalencia del trastorno por déficit de atención-hiperactividad en escolares de una zona urbano-marginal de Lima. Psique Mag. 2017; 6(1): 65 - 71. 
12.Pineda Santos M.A. Trastorno por déficit de atención e hiperactividad en las escuelas de San Pedro Sula, 2016. Tesis doctoral. Universidad Nacional Autónoma de Honduras en el Valle de Sula; 2016. Disponible en: http:// www.bvs.hn/TMVS/pdf/TMVS34/pdf/ TMVS34.pdf

13.Reyes Ticas J.A, Reyes Ochoa E. Trastorno por déficit de atención e hiperactividad (TDAH) en adultos. Rev Med Hond. 2010; 78(4): 196-202. Disponible en: http://www.bvs.hn/RMH/ pdf/2010/pdf/Vol78-4-2010-10.pdf

14.Barragán Pérez E, De la Peña F. Primer Consenso Latinoamericano y declaración de México para el trastorno de déficit de atención e hiperactividad en Latinoamérica. Rev Med Hondur 2008; 76(1): 33-38. Disponible en: http://www.bvs.hn/ $\mathrm{RMH} / \mathrm{pdf} / 2008 / \mathrm{pdf} /$

Vol76-1-2008-9.pdf

15.Balbuena Rivera F. La elevada prevalencia del TDAH: posibles causas y repercusiones socioeducativas. Psicol Educ 22 (2016) 81 - 85. http:// dx.doi.org/10.1016/j.pse.2015.12.002

16.Treviño Cázares $C . D$, Juárez Treviño $M$, González Díaz S.N, Arias Cruz A, Treviño Treviño J.O. Asociación entre el Trastorno por déficit de Atención e Hiperactividad y la Rinitis Alérgica: Una Revisión. Arch de Med. 2015; 11(1:9). doi: 10.3823/1225.

17. Niño Malagón C, Correa Giraldo J, Henríquez Posada N. Estrategias neuropsicopedagógicas para niños $\mathrm{y}$ niñas con bajo rendimiento académico asociado a TDAH. En clave social. 2015; 4(2): $\quad 26 \quad-\quad 38 . \quad \mathrm{http}: / /$ repository.lasallista.edu.co:8080/ojs/ index.php/EN-Clave/article/ viewFile/1038/904
18.Duarte Z.E, Reyes E, Sosa A.L, Risso E, Reyes A. Munguía A. Et al. Prevalencia de TDAH, Relación con Reprobación Escolar y Estado Nutricional en Población Escolar del Distrito Central. Rev Post Med UNAH. 2010; 13(3): 1-10. Disponible en: http:// cidbimena.desastres.hn/RMP/pdf/2010/ pdf/Nol13-3-2010-10.pdf

19. Soria-Claros AM, Serrano I, Serra A, Félix M, Quintero J, Ortiz T. Diferencias neurofuncionales de la onda P300 ante estimulación multisensorial en niños con trastorno por déficit de atención/ hiperactividad. Rev Neurol 2015; 60 (Supl 1): S75-80.

20.Colomer C, Mercader J, Presentación M.J, Miranda A. Influencia de factores familiares y personales en la evolución negativa del TDAH. International Journal of Developmental and Educational Psychology INFAD Revista de Psicología. 2014 4(1). ISSN: 0214-9877. pp:415-424. https://doi.org/10.17060/ ijodaep.2014.n1.v4.629

21.Perea-Martínez A, López-Navarrete G, Carbajal-Rodríguez L, Rodríguez-Herrera $\mathrm{R}$, Zarco-Román J, Loredo-Abdalá $\mathrm{A}$. Alteraciones en la nutrición fetal y en las etapas tempranas de la vida. Su repercusión sobre la salud en edades posteriores. Acta Pediatr Mex.33(1): 26-31. 2012.

22.Pin Arboledas G, Merino Andreu M, Mompo Marabotto M.L. Alteraciones del sueño y TDAH o TDAH y alteraciones del sueño ¿existe relación? Pediatr Integral 2014; XVIII (9): 668-677. Disponible en: https://www.pediatriaintegral.es/ publicacion-2014-11/alteraciones-delsueno-y-tdah-o-tdah-y-alteraciones-delsueno-existe-relacion/

23. Cardo E, Amengual-Gual M. ¿Se asocia el trastorno por déficit de atención/hiperactividad con otras patologías prevalentes de la infancia? 